\title{
Uso de fitoterápicos como coadjuvantes para redução de gordura visceral em pessoas com sobrepeso e/ou obesidade
}

\author{
Use of herbal medicines as adjuvants for reducing visceral fat in overweight and / or obese people \\ Uso de medicamentos a base de hierbas como adyuvantes para la reducción de la grasa visceral en
} personas con sobrepeso y / o obesidad

Recebido: 02/07/2021 | Revisado: 07/07/2021 | Aceito: 13/07/2021 | Publicado: 23/07/2021

Laysse Silva Morais

ORCID: https://orcid.org/0000-0003-1597-6383

Faculdade Estácio de Sá, Brasil

E-mail: layssenutri@hotmail.com

Andrea Nunes Mendes de Brito

ORCID: https://orcid.org/0000-0002-8270-9018

Faculdade Estácio de Sá, Brasil

E-mail: drea.nunes@hotmail.com

Lana Raysa da Silva Araújo

ORCID: https://orcid.org/0000-0003-1224-5788

Universidade Federal do Piauí, Brasil

E-mail: lannaraysa@hotmail.com

Fabiana Neves Lima

ORCID: https://orcid.org/0000-0002-1894-6529

Universidade Federal do Piauí, Brasil

E-mail: fabiananeves@ifpi.edu.br

Gabriela Chamusca Lopes da Silva

ORCID: https://orcid.org/0000-0001-7073-6046 Faculdade Estácio de Sá, Brasil

E-mail: gabriela.chamusca@gmail.com

Daniela Caetano Gonçalves

ORCID: https://orcid.org/0000-0002-0604-7067 Faculdade Estácio de Sá, Brasil

E-mail: tccnutcoordenador@gmail.com

Ana Lúcia Hoefel

ORCID: https://orcid.org/0000-0003-0940-3263 Faculdade Estácio de Sá, Brasil

E-mail: tccnutcoordenador@gmail.com

\begin{abstract}
Resumo
O objetivo deste estudo foi investigar se a utilização de fitoterápicos como coadjuvantes ao tratamento nutricional pode auxiliar no controle da gordura visceral em pessoas com sobrepeso/obesidade. No desenvolvimento da pesquisa realizou-se uma revisão integrativa cuja questão focal foi avaliar a eficácia dos fitoterápicos que são utilizados no tratamento nutricional para controle da gordura visceral. As buscas foram realizadas nas bases eletrônicas de dados, entre os meses de setembro de 2018 a março de 2019. De acordo com os estudos encontrados, as maiores abordagens contemplaram a utilização da camellia sinensis (chá verde) e suas catequinas. Contudo, também foram analisadas pesquisas a respeito do óleo de canola, páprica xantofila, garcínia cambogia, café verde, ácidos linoleico, linolênico e esteárico, dentre outros. Os resultados apontam efeitos positivos entre as pesquisas, o que caracteriza a fitoterapia como uma importante estratégia nutricional no combate à gordura visceral e excesso de peso, principalmente quando aliada à reeducação alimentar e prática de atividade física regular.
\end{abstract}

Palavras chave: Fitoterápicos; Coadjuvante; Gordura visceral; Sobrepeso; Obesidade.

\begin{abstract}
The objective of this study was to investigate whether the use of herbal products as adjuncts to nutritional treatment may help to control visceral fat in overweight / obese individuals. In the development of the research, an integrative review was conducted whose focal issue was to evaluate the efficacy of herbal medicines that are used in the nutritional treatment to control visceral fat. The searches were carried out in the electronic databases, from September 2018 to March 2019. According to the studies found, the major approaches included the use of camellia sinensis (green tea) and its catechins. However, research was also carried out on canola oil, xanthophyll paprika, garcinia cambogia, green coffee, linoleic, linolenic and stearic acids, among others. The results point to positive effects among
\end{abstract}


the researches, which characterizes phytotherapy as an important nutritional strategy in the fight against visceral fat and overweight, especially when combined with dietary reeducation and regular physical activity.

Keywords: Phytotherapics; Support; Visceral fat; Overweight; Obesity.

\section{Resumen}

El objetivo de este estudio fue investigar si el uso de medicamentos a base de hierbas como complemento del tratamiento nutricional puede ayudar a controlar la grasa visceral en personas con sobrepeso / obesidad. En el desarrollo de la investigación se realizó una revisión integradora cuya pregunta focal fue evaluar la efectividad de los medicamentos herbales que se utilizan en el tratamiento nutricional para el control de la grasa visceral. Las búsquedas se realizaron en bases de datos electrónicas, entre los meses de septiembre de 2018 y marzo de 2019. Según los estudios encontrados, los mayores enfoques contemplaron el uso de camellia sinensis (té verde) y sus catequinas. Sin embargo, también se analizaron investigaciones sobre aceite de canola, xantofila de pimentón, garcinia cambogia, café verde, ácidos linoleico, linolénico y esteárico, entre otros. Los resultados apuntan a efectos positivos entre los estudios, lo que caracteriza a la fitoterapia como una importante estrategia nutricional para combatir la grasa visceral y el exceso de peso, especialmente cuando se combina con reeducación dietética y actividad física regular.

Palabras clave: Medicamentos a base de plantas; Secundario; Grasa visceral; Exceso de peso; Obesidad.

\section{Introdução}

A adiposidade abdominal e os distúrbios metabólicos associados definem o que se considera síndrome metabólica. Esta, se apresenta como uma epidemia mundial que tem contribuído para o crescimento de morbimortalidade populacional (Diniz et al., 2009).

Pitanga et al., (2012) definem gordura visceral como a quantidade de gordura localizada na região intra-abdominal. Para sua mensuração, a medida da circunferência da cintura é o método mais comumente utilizado, havendo sugestões de pontos de corte associados ao maior risco cardiovascular (Ribeiro Filho et al., 2006).

Os pontos mais utilizados são os recomendados pelas diretrizes brasileiras de obesidade (Abeso, 2016), em que a medida igual ou superior a $94 \mathrm{em}$ homens e $80 \mathrm{~cm}$ em mulheres, é indicativa para a incidência de desordens metabólicas.

Nos últimos anos, algumas modificações nos hábitos alimentares e comportamentais da população como: sedentarismo, história familiar e ingestão alimentar insatisfatória, contribuíram para o acúmulo calórico diário e consequente crescimento do índice de obesidade dos indivíduos (Conceição et al., 2018).

Este fato, fez com que a população buscasse alternativas de combate ao sobrepeso/obesidade, dentre elas, o uso de fitoterápicos ou plantas medicinais tem se destacado. A justificativa para tal fato estaria no avanço das pesquisas cientificas, que tem comprovado a segurança e eficácia dos mesmos. Bem como, se comparado as terapias convencionais, os fitoterápicos são considerados terapias menos agressivas (com pouco ou nenhum efeito colateral) e de baixo custo (Bruning et al., 2012; Verrengia et al., 2013).

Segundo a RDC N 26/2014, são considerados fitoterápicos os obtidos com emprego exclusivo de matérias-primas ativas vegetais, cuja segurança e eficácia sejam baseadas em evidências clínicas e que sejam caracterizados pela constância de sua qualidade. Inclusive, quando associados ao tratamento dietético, podem aumentar a eficácia das intervenções nutricionais devido a ação dos seus compostos ativos (Brasil, 2014; Silveira et al., 2012).

Contudo, apresentam dentre outras vantagens, a facilidade de acesso, visto que, o produto pode ser adquirido em diferentes formas: industrializado, manipulado ou mesmo mediante ao uso das plantas, em preparações caseiras (Figueiredo et al., 2014).

Considerando que a obesidade é um grave problema de saúde pública e que necessita de diferentes formas de tratamento para seu combate, o objetivo deste estudo foi investigar se a utilização de fitoterápicos como coadjuvantes ao tratamento nutricional pode auxiliar no controle da gordura visceral em pessoas com sobrepeso/obesidade. 


\section{Metodologia}

Este estudo trata-se de uma revisão integrativa cuja questão focal é: Qual a eficácia dos fitoterápicos utilizados no tratamento nutricional para controle da gordura visceral?

As buscas foram realizadas nas bases eletrônicas de dados LILACS, PUBMED e BIREME, entre setembro de 2018 e março de 2019, utilizando como critério de inclusão artigos originais, (experimentais, ensaio clínico), publicados no período de 2008 e 2018 (10 anos), de idioma inglês e português. Os descritores usados foram "fitoterapia, gordura abdominal, sobrepeso e obesidade", e seus equivalentes em inglês.

Foram adotados como critérios de exclusão artigos de revisão, artigos repetidos, relatos de casos ou séries de casos, cartas ao editor e artigos que não condizem com a questão focal, teses e dissertações.

Os artigos identificados durante a busca nas bases de dados foram selecionados a partir da leitura dos títulos, seguido dos resumos. Após esta etapa, os estudos foram lidos na integra. As referências dos estudos selecionados foram rastreadas, com vistas à inclusão de outros artigos de potencial interesse.

O procedimento foi feito por dois pesquisadores simultaneamente e de forma independente, levando-se em consideração os critérios de inclusão e exclusão pré-definidos. Os trabalhos para o qual houve discordância foram analisados em reunião com os autores para avaliação e consenso sobre a inclusão na revisão.

A extração dos dados foi realizada por meio de um protocolo elaborado pelos pesquisadores, no qual foram incluídos os seguintes dados: título, metodologia, principais resultados e conclusão.

\section{Resultados e Discussão}

Esta revisão agrupou estudos que relacionavam plantas medicinais ou fitoterápicos ao tratamento do sobrepeso/obesidade e em específico, gordura visceral, também chamada na literatura de gordura abdominal ou obesidade central. Foram encontrados 15 estudos considerados relevantes, conforme a Tabela 1.

Tabela 1. Estudos de fitoterápicos relacionados com a gordura visceral.

\begin{tabular}{|c|c|c|c|}
\hline Autor/Ano & Metodologia & Resultados & Conclusão \\
\hline $\begin{array}{c}\text { Sasaki e } \\
\text { colaboradores, } 2013\end{array}$ & $\begin{array}{c}\text { Foram suplementados } 24 \text { ratos por } \\
62 \text { dias com ácido linoleico } \\
\text { conjugado }\end{array}$ & $\begin{array}{l}\text { Os ratos suplementados reduziram } \\
\text { a gordura corporal se comparados } \\
\text { ao grupo controle }\end{array}$ & $\begin{array}{l}\text { Observou-se que a suplementação foi } \\
\text { eficiente tanto para a redução da } \\
\text { gordura corporal total, quanto para a } \\
\text { gordura visceral dos animais. }\end{array}$ \\
\hline $\begin{array}{c}\text { Wang e } \\
\text { colaboradores, } 2010\end{array}$ & $\begin{array}{l}182 \text { indivíduos com excesso de } \\
\text { peso foram divididos em grupos e } \\
\text { consumiram doses diferenciadas } \\
\text { de catequinas por } 90 \text { dias. Os } \\
\text { dados foram coletados aos } 0,30 \text {, } \\
60 \text { e } 90 \text { dias. }\end{array}$ & $\begin{array}{l}\text { Houve uma diminuição na área } \\
\text { de gordura intra-abdominal de } 5,6 \\
\mathrm{~cm} \text { no grupo que ingeriu dose extra } \\
\text { alta de catequina. Além disso, foi } \\
\text { encontrado decréscimos de } 1,9 \mathrm{~cm} \\
\text { na circunferência da cintura e } 1,2 \\
\text { kg no grupo que ingeriu dose extra } \\
\text { alta de catequina versus controle. }\end{array}$ & $\begin{array}{l}\text { O consumo de duas porções de uma } \\
\text { catequina extra alta leva a melhorias na } \\
\text { composição corporal e reduz a gordura } \\
\text { abdominal em indivíduos } \\
\text { moderadamente acima do peso. }\end{array}$ \\
\hline $\begin{array}{c}\text { Oliveira e } \\
\text { Colaboradores, } 2017\end{array}$ & $\begin{array}{l}\text { Foram avaliados os fitoterápicos } \\
\text { Camelia sinensis, } \\
\text { Citrus aurantium e faseolamina. } \\
\text { Uma amostra composta por } 77 \\
\text { pacientes, de ambos os sexos e } \\
\text { adultos durante } 8 \text { semanas. }\end{array}$ & $\begin{array}{c}\text { Foi observada maior redução de } \\
\text { gordura corporal no grupo tratado } \\
\text { com Camelia sinensis, com perda } \\
\text { de } 0,85 \% \text { de gordura corporal, } \\
\text { comparado à faseolamina }(0,81 \%), \\
\text { Citrus }(0,68 \%) \text { e grupo controle } \\
(0,34 \%) .\end{array}$ & $\begin{array}{l}\text { Os fitoterápicos em paralelo à } \\
\text { reeducação alimentar apresentaram } \\
\text { resultados positivos em relação à perda } \\
\text { de peso e redução dos níveis de gordura } \\
\text { corporal. }\end{array}$ \\
\hline
\end{tabular}


Maia-Landim e colaboradores, 2018.

Saito e

colaboradores, 2016.

Kakutani e colaboradores, 2018

Kim e Park, 2015.

Oliveira-De-Lira e colaboradores, 2018.

Shen e

Colaboradores, 2014.

Sugita e colaboradores, 2014

Raso e colaboradores, 2017.
214 pessoas com

sobrepeso ou obesidade foram submetidos a uma dieta controla suplementada com Garcínia

Cambogia e Glucomanano por 6 meses.
O tratamento produziu perda de peso, reduzindo a massa gorda, a gordura visceral, os lipídios e os níveis de glicose no sangue, além de aumento da taxa metabólica basal.
A administração de Garcínia cambogia

e Glucomanano reduz o peso e melhora o perfil bioquímico de pessoas com sobrepeso ou obesidade, embora a presença de polimorfismos PLIN4, FTO e ADRB3 possa dificultar em algum grau esses efeitos.

A redução na gordura visceral foi significativamente maior no grupo ALA-DAG quando comparado ao grupo TAG. O peso corporal e a circunferência da cintura, também foram significativamente menores no grupo ALA-DAG do que no grupo TAG.

No grupo da xantofila páprica, a redução da área de gordura

sobrepeso ou obesidade foram submetidos a uma dieta controla suplementada com Garcínia

Cambogia e Glucomanano por 6 meses.

subcutânea, área de gordura total e

IMC foi significativamente maior quando comparado ao grupo placebo.

Camundongos que receberam uma dieta rica em gordura com suco de pimenta verde, tiveram uma diminuição de gordura visceral em comparação com o grupo controle, além de menor ganho de peso.

O óleo de coco deve

75 mulheres foram divididas em grupos e suplementadas com diferentes tipos de óleos: óleo de coco, cártamo, chia e o placebo de óleo de soja. $\mathrm{O}$ experimento durou 8 semanas.

maior efeito sobre a adiposidade abdominal e perfil glicídico, enquanto o óleo de chia teve um efeito

de melhora do perfil lipídico.

O peso corporal total não diferiu significativamente entre os grupos alimentares. No entanto, a gordura visceral foi reduzida em $70 \%$ no grupo alimentado com ácido esteárico em comparação com outras dietas.

A ingestão diária de grãos do paraíso (GP) aumentou o gasto energético corporal e diminuiu a área de gordura visceral.

Não houve diferença significativa no peso corporal entre os grupos. Porém, quanto à gordura visceral, o grupo do chá verde apresentou área menor ao final do experimento.
Em conclusão, o ALA-DAG pode ser útil para reduzir a AGV (área da gordura visceral) e concomitantemente suprimir o ganho de peso sem efeitos colaterais.

Houve redução significativa da área de voluntários saudáveis com sobrepeso, sem causar efeitos adversos.

Resultados sugerem que o suco de pimenta verde, como uma bebida, pode ser útil na redução do ganho de peso e controle dos níveis de lipídios séricos.

De fato, a suplementação com diferentes composições de ácidos graxos resultou

em respostas positivas e específicas, quando associada a uma dieta hipocalórica.

O ácido esteárico dietético leva a uma redução drástica da gordura visceral, provavelmente causando a apoptose dos pré-adipócitos.

O extrato GP pode ser uma ferramenta eficaz e segura para reduzir a gordura corporal, principalmente por prevenir o acúmulo de gordura visceral.

O consumo crônico de chá verde leva à diminuição da área do tecido adiposo visceral. gordura abdominal e o IMC em 


\begin{tabular}{|c|c|c|c|}
\hline $\begin{array}{c}\text { Heber e } \\
\text { colaboradores, } 2014 .\end{array}$ & $\begin{array}{l}\text { Os ratos foram alimentados com } \\
\text { dietas obesogênicas, onde apenas } \\
\text { um dos grupos recebeu adição de } \\
\text { extrato de polifenóis }(0,25 \%) \text {. O } \\
\text { experimento durou } 20 \text { semanas. }\end{array}$ & $\begin{array}{l}\text { No grupo que que ingeriu o extrato } \\
\text { de polifenóis, houve diminuição do } \\
\text { peso corporal, volume de gordura } \\
\text { visceral e dos biomarcadores de } \\
\text { inflamação. }\end{array}$ & $\begin{array}{l}\text { O tratamento com polifenóis, a longo } \\
\text { prazo, atenuou o desenvolvimento de } \\
\text { obesidade, sintomas associados à } \\
\text { síndrome metabólica e esteatose } \\
\text { hepática. }\end{array}$ \\
\hline $\begin{array}{c}\text { Song, Choi e Park, } \\
2014 .\end{array}$ & $\begin{array}{l}48 \text { ratos foram divididos em } \\
\text { grupos e suplementados com } \\
\text { dietas: hiperlipídica, com extrato } \\
\text { de café verde descafeinado e ácido } \\
\text { 5-cafeoilquínico em diferentes } \\
\text { concentrações, durante } 11 \\
\text { semanas. }\end{array}$ & $\begin{array}{c}\text { O extrato de café verde } \\
\text { descafeinado mostrou efeito } \\
\text { positivo na redução de peso em } \\
\text { camundongos alimentados com } \\
\text { dieta hiperlípidica e com dose } \\
\text { mínima de } 0,3 \% \text { de suplementação. }\end{array}$ & $\begin{array}{l}\text { O extrato de grão de café verde reduz } \\
\text { significativamente o acúmulo de } \\
\text { gordura visceral e melhora a resistência } \\
\text { à insulina em camundongos. }\end{array}$ \\
\hline
\end{tabular}

Fonte: Pesquisa direta.

As abordagens mais observadas foram sobre a camellia sinensis e suas catequinas, as principais evidencias sugerem uma relação entre a redução dos níveis hepáticos de LPL e a inflamação na obesidade, inclusive quando associada a outros fitoterápicos como faseolamina e citrus aurantium (Oliveira et al., 2017 e Nam et al., 2018).

Também segundo Wang et al., (2010), houve relação do chá verde (camellia sinensis) com a melhora da composição corporal e redução da gordura abdominal, ao comparar diferentes níveis de dosagens e suas interações.

O principal polifenol do chá verde, epigalocatequina-3-galato (EGCG) teve seus efeitos analisados por Bose et al., (2008), através de um estudo experimental, em que os camundongos foram alimentados com dieta induzida por gordura e suplementados com epigalocatequina-3-galato por 16 semanas. Os resultados evidenciaram redução do percentual de gordura corporal e do peso da gordura corporal visceral.

Em um levantamento bibliográfico realizado por Uemoto \& Coimbra, (2013), com 56 artigos sobre o seguinte tema: analisar se o chá verde (camellia sinensis) era eficaz no tratamento da obesidade. Observou-se que devido a seu efeito termogênico resultante da interação das catequinas, principalmente a epigalocatequina galato e a cafeína, o chá verde pode ser considerado uma alternativa para o tratamento da obesidade, desde que associado a reeducação alimentar e exercícios físicos regulares.

Contudo, Hibi et al., (2018), realizaram um ensaio clínico, onde analisou-se paralelamente a eficácia das catequinas do chá verde para a redução do risco de síndrome metabólica associada à redução da gordura abdominal. Os dados foram agrupados a partir de seis ensaios em humanos durante 12 semanas. Os resultados evidenciaram o que Bose et al., (2008) e Uemoto \& Coimbra, (2013) afirmaram acima em seu experimento e levantamento bibliográfico, respectivamente. Isto reforça a tese de que o chá verde pode ser um importante aliado para o combate da gordura visceral.

Sobre os ácidos graxos, foram encontrados na literatura os ácidos: linolênico, linoleico e oleico, também chamados de ômegas 3, 6, 9 respectivamente. No estudo clínico de Liu et al., (2016) foi realizado um ensaio clínico que comparou os efeitos da canola com o óleo de canola de alto teor oleico. Os resultados mostraram que o consumo em curto prazo de dietas ricas em ácidos graxos monoinsaturados, fornecidos pelo óleo de canola, foi associado a uma redução da adiposidade abdominal e, portanto, considerado eficaz para o tratamento de síndrome metabólica.

O ácido linoleico foi analisado de forma experimental, onde 24 ratos foram suplementados por 62 dias, os resultados mostraram resultado similar ao citado anteriormente, redução da gordura corporal e visceral (Sazaki et al., 2013). O ácido linoleico conjugado é considerado um potente agente antiobesidade pelas suas propriedades moduladoras do metabolismo lipídico. Além do que, tem se mostrado eficaz para a diminuição da gordura corporal em estudos experimentais (Turchetto et al., 2013). 
Em contrapartida, o ácido esteárico, apesar de ser um ácido graxo saturado, também demonstrou ser um aliado na redução de gordura visceral. Mas, é importante ressaltar, que o estudo encontrado de Shen et al., (2014) apresentou algumas limitações, como por exemplo, não provar que o ácido esteárico dietético de forma isolada, causou a redução no tecido adiposo visceral e que as dietas de óleo de milho e óleo de cártamo continham ácidos graxos em grande parte na forma de triglicerídeos, enquanto a dieta com ácido esteárico usava ácido esteárico não esterificado.

Com relação à garcinia cambogia, a literatura diz que é um fruto encontrado em uma planta da família Guttiferácias (clusiáceas), nativa da Ásia, utilizada antigamente com função de conservante e aromatizante. Seu princípio ativo é encontrado na casca do fruto, ácido hidroxicítrico (AHC), que parece ter ação hipolipemiante, podendo ser usado no tratamento da obesidade (Rosa \& Machado, 2016).

No estudo de Maia-Landim et al., (2018), foram suplementados Garcínia Cambogia e Glucomanano em pessoas com sobrepeso ou obesidade por 6 meses. Os resultados mostraram que houve redução de peso, gordura corporal e visceral. Em sumo, os estudos mostram tendência para um efeito positivo, da utilização da Garcinia cambogia sobre a obesidade, com pouca possibilidade de efeitos colaterais. Porém, são necessários mais ensaios clínicos randomizados e com um maior tempo de intervenção para melhor segurança e aplicação terapêutica.

Outro estudo encontrado na literatura, diz respeito a páprica - xantófila. A Páprica é um pó de coloração vermelha obtido pela moagem de frutos desidratados de pimentão (Capsicum annиит), é considerada um dos condimentos mais consumidos no mundo. Somente os frutos completamente maduros, de coloração vermelha intensa e de alta qualidade são usados para sua produção (Ribeiro, 2012).

Kakutani et al., (2018) administraram, por via oral, cápsulas de páprica xantófila em 100 voluntários saudáveis durante 12 semanas e comparou com o grupo controle. No grupo da páprica xantófila, a redução da área de gordura subcutânea, área de gordura total e IMC foi significativamente maior quando comparado ao grupo placebo. Os resultados sugeriram que a páprica xantófila é um ingrediente alimentar seguro que melhora o metabolismo lipídico e reduz a gordura abdominal.

Nos resultados encontrados, também se ressaltou a Aframomum melegueta (espécie da família do gengibre), através de estudo controlado que mediu a gordura corporal antes e após a ingestão do extrato alcoólico de grãos do paraíso (Aframomum melegueta), sugerindo que também pode ser considerada uma ferramenta eficaz e segura para reduzir a gordura corporal, principalmente pela prevenção do acúmulo de gordura visceral (Sugita et al., 2014).

Outro experimento a apresentar destaque, foi a respeito do extrato de café verde (coffea robusta). Song et al., (2014) suplementaram camundongos com diferentes concentrações do extrato de café verde por 11 semanas e, verificaram que com concentração mínima de $0,3 \%$ é possível observar efeito positivo na redução de peso e volume de gordura visceral.

Este efeito positivo do café verde é justificado por conter uma rica fonte de polifenóis, como o ácido clorogênico, citado na literatura como atuante e protetor do estresse oxidativo, metabolismo da glicose e doenças cardiovasculares (Budryn et al., 2017).

Portanto, é evidente que a fitoterapia tem mostrado destaque em meio aos impactos negativos causados pela síndrome metabólica (SM) e o crescente aumento da gordura visceral, considerada seu principal marcador. A busca por métodos diagnósticos e de intervenção precoce da SM são importantes aliados para a garantia de qualidade de vida das pessoas (Diniz et al., 2009).

\section{Considerações Finais}

Pode-se observar que os estudos com relação a fitoterápicos ainda se caracterizam como recentes, principalmente quando se relaciona a gordura visceral. Os resultados desta pesquisa bibliográfica evidenciaram a relação positiva da Camellia 
Sinensis e de alguns ácidos graxos com a gordura visceral, fitoterápicos encontrados com maior frequência na literatura e, portanto, com maior número de achados clínicos.

Porém, é importante destacar que por ser algo novo, a maior parte dos estudos são de natureza experimental. Portanto, são necessárias mais pesquisas randomizadas e ensaios clínicos, com períodos de análise maiores para que se possa ter segurança na prescrição e dosagens destes fitoterápicos.

Ressalta-se ainda que nenhum tratamento quando praticado de forma isolada é suficiente para controle de sobrepeso/obesidade. É preciso que haja uma sinergia dos mais diversos parâmetros, tendo como base a reeducação alimentar e atividade física associadas, para uma maior efetividade de qualquer estratégia nutricional.

\section{Referências}

Diniz, A. L. D., Tomé, R. A. F., Debs, C. L., Carraro, R, Roever, L. B. \& Pinto, R. M. C. (2009). Avaliação da reprodutibilidade ultrassonográfica como método para medida da gordura abdominal e visceral. Radiol. Bras. 42(6):353-357. https://doi.org/10.1590/S0100-39842009000600007.

Pitanga, C. P. S., Pitanga, F. J. G., Beck, C. C., Gabriel, R. E. C. D. \& Moreira, M. H. R. (2012). Nível de atividade física para prevenção do excesso de gordura visceral em mulheres pós menopáusicas: quanto é necessário? Arq. Bras. Endocrinol. Metab. 56(6): 358-363. https://doi.org/10.1590/S000427302012000600003 .

ABESO, Associação Brasileira para o Estudo da Obesidade e da Síndrome Metabólica (2016). Diretrizes brasileiras de obesidade. (4a ed.).

Conceição, F. R., Gomes, V. T. S., Nina, M. L. N., Pedrosa, N. O., Souza, I. R., Santos, A. T. S. \& Barbosa, F. E. (2018). Terapia complementar: A comercialização de fitoterápicos para o controle do peso em um município do Maranhão. Revista Eletrônica Acervo Saúde, 10 (S1030-S1036). 10.25248/REAS188_2018.

Bruning, M. C. R., Mosegui, G. B. G. \& Vianna, C. M. M. (2012). A utilização da fitoterapia e de plantas medicinais em unidades básicas de saúde nos municípios de Cascavel e Foz do Iguaçu - Paraná: a visão dos profissionais de saúde. Ciência \& Saúde Coletiva, 17(10):2675-2685. https://doi.org/10.1590/S1413-81232012001000017.

Verrengia, E. C., Kinoshita, S. A. T., \& Amadei, J. L. (2013). Medicamentos Fitoterápicos no Tratamento da Obesidade. Uniciências, 17 (1); $53-58$.

BRASIL, Ministério da Saúde (2014). Resolução da diretoria colegiada $\quad$ - RDC $n^{\circ} 26$, de $13 \quad$ de maio de 2014 . http://bvsms.saude.gov.br/bvs/saudelegis/anvisa/2014/rdc0026_13_05_2014.pdf.

Vieira, A. R. R. \& Medeiros, P. R. M. S. (2019). A utilização de fitoterápicos no tratamento da obesidade. Rev Cient Esc Estadual Saúde Pública Goiás "Cândido Santiago";5(1):44-57.

Figueredo, C. A., Gurgel, I. G. D. \& Gurgel Junior, G. D. (2014). A Política Nacional de Plantas Medicinais e Fitoterápicos: construção, perspectivas e desafios. Revista de Saúde Coletiva, 24 (2). https://doi.org/10.1590/S0103-73312014000200004.

Oliveira, A. P., Bernardes, A. C. B., Fernandes, F. L. F. \& Tiengo, A. (2017). Avaliação dos efeitos de fitoterápicos termogênicos em parâmetros antropométricos de pacientes com sobrepeso e obesidade. RBONE-Revista Brasileira de Obesidade, Nutrição e Emagrecimento, 11(.68); 667-676.

Nan, M., Choi, M. S., Choi, J. Y., Kim, N., Kim, M. S. \& Jung, S. (2018). Effect of green tea on hepatic lipid metabolism in mice fed a high-fat diet. The Journal of Nutritional Biochemistry. 51: 1-7. 10.1016 / j.jnutbio.2017.09.002.

Wang, H., Wen, Y., Du, Y., Yan, X., Guo, H., Rycroft, J. Á., \& Mela, D. J. (2010). Effects of Catechin Enriched Green Tea on Body Composition. Obesity journal 18, 773-779. 10.1038/oby.2009.256.

Hibi, M., Takase, H., Iwasaki, M., Osaki, N. \& Katsuragi, Y. (2018). Efficacy of tea catechin-rich beverages to reduce abdominal adiposity and metabolic syndrome risks in obese and overweight subjects: a pooled analysis of 6 human trials. Nutr Res. 55; 1-10. 10.1016/j.nutres.2018.03.012.

Bose, M., Lambert, J. D., Ju, J., Reuhl, K. R., Shapses, A. S. \& Yang, C. S. (2008). The major green tea polyphenol, (-)-epigallocatechin-3-gallate, inhibits obesity, metabolic syndrome, and fatty liver disease in high-fat-fed mice. The journal of nutrition, 138 (9).

Uemoto, M. Y. \& Coimbra, C. C. B. E. (2013). A utilização da camellia sinensis na prevenção e tratamento da obesidade. Revista Uningá Review, 16 (2).

Liu, X., Kris-Etherton, P. M., West, S. G., Lamarche, B., Jenkins, D. J. A. \& Fleming, J. A., Jones, P. J. H. (2016). Effects of canola and high-oleic-acid canola oils on abdominal fat mass in individuals with central obesity. Obesity Symposium. Clinical Trials and Investigations. 24 (11): 2261-2268. 10.1002 / oby. 21584 .

Sasaki, C. A. L., Santos, J. O., Nogueira, J. A. D., Fontana, K. E. \& Oliveira, R. J. (2013). Efeito da suplementação oral com ácido linoleico conjugado associado ao treinamento físico sobre a gordura corporal de ratos. Rev. educ. fis. UEM, 24 (1);103-109, 1. https://doi.org/10.4025/reveducfis.v24.1.16865

Turchetto, Q., Andreato, L. V., Almeida, F. N., Esteves, J. V. D. C. \& Moraes, S. M. F. (2013). Suplementação de ácido linoleico conjugado associado ao exercício físico em parâmetros morfofuncionais de ratos submetidos à dieta de cafeteria. Rev. educ. fis. UEM; 24 (1): 121-134, 1. https://doi.org/10.4025/reveducfis.v24.1.15196 
Research, Society and Development, v. 10, n. 9, e15610917831, 2021

(CC BY 4.0) | ISSN 2525-3409 | DOI: http://dx.doi.org/10.33448/rsd-v10i9.17831

Shen, M. C., Zhao, X., Siegal, G. P., Desmond, R. \& Hardy, R. W. (2014). Dietary stearic acid leads to a reduction of visceral adipose tissue in athymic nude mice. PLoS One. 2014;9(9):e104083. 10.1371/journal.pone.0104083

Rosa, F. M. M. \& Machado, J. T. (2016). O efeito anti-obesidade da Garcinia cambogia em humanos. Revista Fitos. 10(2), 95-219, Abr-Junho. 10.5935/24464775.20160014

Maia- Landim, A., Ramírez, J. M., Lancho, C., Poblador, M. S. \& Lancho, J. L. (2018). Long-term effects of Garcinia cambogia/Glucomannan on weight loss in people with obesity, PLIN4, FTO and Trp64Arg polymorphisms. BMC Complementary and Alternative Medicine, 18: 26. 10.1186 / s12906-018-2099-7

Ribeiro, C. S. C. (2012). Qualidade de páprica. In: Congresso Brasileiro de Olericultura, 52. Horticultura Brasileira, 30(2).

Kakutani, R., Hokari, S., Nishino, A., ... \& Maoka, T. (2018). Effect of Oral Paprika Xanthophyll Intake on Abdominal Fat in Healthy Overweight Humans: A Randomized, Double-blind, Placebo-controlled Study. J Oleo Sci; 67(9):1149-1162. 10.5650/jos.ess 18076.

Sugita, J., Yoneshiro, T., Sugishima, Y., Ikemoto, T., Uchiwa, H., Suzuki, I. \& Saito, M. (2014). Daily ingestion of grains of paradise (Aframomum melegueta) extract increases whole-body energy expenditure and decreases visceral fat in humans.J Nutr Sci Vitaminol (Tokyo); 60(1):22-27. $10.3177 /$ jnsv. 60.22

Song, S. J., Choi, S. \& Park, T. (2014). Decaffeinated green coffee bean extract attenuates diet-induced obesity and insulin resistance in mice. Evid Based Complement Alternat Med; 2014:718379. 10.1155/2014/718379

Brudryn, G., Zaczyńska, D., Żyżelewicz, D., Grzelczyk, J., Zduńczyk, Z. \& Juśkiewicz, J. (2017). Influence of the Form of Administration of Chlorogenic Acids on Oxidative Stress Induced by High fat Diet in Rats. Plant Foods Hum Nutr.72:184-191.

Ribeiro Filho, F. F. R., Mariosa, L. S., Ferreira, S. R. G. \& Zanella, M. T. (2006). Gordura Visceral e Síndrome Metabólica: Mais Que Uma Simples Associação. Arq Bras Endocrinol Metab; 50 (2). https://doi.org/10.1590/S0004-27302006000200009

Heber, D., Zhang, Y., Yang, J., Ma, J. E., Henning, S. M. \& Li, Z. (2014). Green tea, black tea, and oolong tea polyphenols reduce visceral fat and inflammation in mice fed high-fat, high-sucrose obesogenic diets. J Nutr., 144 (9): 1385-93. 10.3945 / jn.114.191007.

Kim, N. H. \& Park, S. H. (2015). Evaluation of green pepper (Capsicum annuum L.) juice on the weight gain and changes in lipid profile in C57BL/6 mice fed a high-fat diet. J Sci Food Agric.;95(1):79-87. 10.1002/jsfa.6685

Oliveira-de-Lira, L., Santos, E. M. C., Souza, R. F., Matos, R. J. B., Silva, M. C. D., Oliveira, L. D. S., Nascimento, T. G., Schemly, P. A. S. \& Souza, S. L. (2018). Supplementation-Dependent Effects of Vegetable Oils with Varying Fatty Acid Compositions on Anthropometric and Biochemical Parameters in Obese Women. Nutrients, 20;10(7):932. 10.3390/nu10070932.

Raso, R. A., Paim, R. R. B., Pinheiro, S. V. B., Tavares Júnior, W. C., Vasconcellos, L. S. \& Alberti, L. R. (2017). Effects of chronic consumption of green tea on weight and body fat distribution of Wistar rats evaluated by computed tomography. Acta Cir Bras.;32(5):342-349.

Saito, S., Fukuhara, I., Osaki, N., Nakamura, H. \& Katsuragi, Y. (2016). Consumption of alpha-Linolenic Acid-enriched Diacylglycerol Reduces Visceral Fat Area in Overweight and Obese Subjects: a Randomized, Double-blind Controlled, Parallel-group Designed Trial. J Oleo Sci. 65(7):603-611. 10.5650/jos.ess 16059 